\title{
Macronutrient intakes and serum oestrogen, and interaction with polymorphisms in CYP19A1 and HSD17B1 genes: a cross-sectional study in postmenopausal Japanese women
}

Sahoko Takagi ${ }^{1}$, Mariko Naito ${ }^{1}$, Sayo Kawai ${ }^{1}$, Rieko Okada $^{1}$, Chisato Nagata $^{2}$, Satoyo Hosono ${ }^{3}$, Yuichiro Nishida ${ }^{4}$, Naoyuki Takashima ${ }^{5}$, Sadao Suzuki ${ }^{6}$, Ippei Shimoshikiryo ${ }^{7}$, Haruo Mikami ${ }^{8}$, Hirokazu Uemura9, Nagato Kuriyama ${ }^{10}$, Keizo Ohnaka ${ }^{11}$, Michiaki Kubo ${ }^{12}$, Nobuyuki Hamajima ${ }^{13}$, Hideo Tanaka ${ }^{3,14}$ and Kenji Wakai ${ }^{1}$ for the Japan Multi-Institutional Collaborative Cohort (J-MICC) Study Group

${ }^{1}$ Department of Preventive Medicine, Nagoya University Graduate School of Medicine, 65 Tsurumai-cho, Showa-ku, Nagoya 466-8550, Japan

${ }^{2}$ Department of Epidemiology and Preventive Medicine, Gifu University Graduate School of Medicine, 1-1 Yanagido, Gifu 501-1194, Japan

${ }^{3}$ Division of Epidemiology and Prevention, Aichi Cancer Center Research Institute, 1-1 Kanokoden, Chikusa-ku, Nagoya 464-8681, Japan

${ }^{4}$ Department of Preventive Medicine, Faculty of Medicine, Saga University, Nabeshima 5-1-1, Saga 849-8501, Japan

${ }^{5}$ Department of Health Science, Shiga University of Medical Science, Seta Tsukinowa-cho, Otsu 520-2192, Japan

${ }^{6}$ Department of Public Health, Nagoya City University Graduate School of Medical Sciences, 1 Kawasumi Mizuho-cho, Mizuho-ku, Nagoya 467-8601, Japan

${ }^{7}$ Department of International Island and Community Medicine, Kagoshima University Graduate School of Medical and Dental Sciences, 8-35-1 Sakuragaoka, Kagoshima 890-8544, Japan

${ }^{8}$ Chiba Cancer Center, Division of Cancer Registry, Prevention and Epidemiology, 666-2 Nitona-cho, Chuo-ku, Chiba 260-0801, Japan

${ }^{9}$ Department of Preventive Medicine, Institute of Biomedical Sciences, Tokushima University Graduate School, 3-18-15,

Kuramoto-cho, Tokushima 770-8503, Japan

${ }^{10}$ Department of Epidemiology for Community Health and Medicine, Kyoto Prefectural University of Medicine, 465 Kajii-cho, Kawaramachibirokoji, Kamigyo-ku, Kyoto 602-8566, Japan

${ }^{11}$ Department of Geriatric Medicine, Graduate School of Medical Sciences, Kyushu University, 3-1-1 Maidashi, Higashi-ku, Fukuoka 812-8582, Japan

${ }^{12}$ Center for Integrative Medical Sciences, RIKEN, 1-7-22 Suehiro-cho, Tsurumi-ku, Yokohama 230-0045, Japan

${ }^{13}$ Department of Healthcare Administration, Nagoya University Graduate School of Medicine, 65 Tsurumai-cho, Showa-ku, Nagoya 466-8550, Japan

${ }^{14}$ Department of Epidemiology, Nagoya University Graduate School of Medicine, 65 Tsurumai-cho, Showa-ku, Nagoya 466-8550, Japan

(Submitted 19 January 2017 - Final revision received 21 July 2017 - Accepted 1 August 2017)

\section{Abstract}

Although higher circulating levels of oestrogen are related to postmenopausal breast cancer risk, limited information is available regarding effects of diet on endogenous oestrogen. Thus, we examined associations between macronutrient intakes and serum oestrogen with consideration of polymorphisms in oestrogen-metabolising genes. In this cross-sectional study, 784 naturally menopaused Japanese women aged 47-69 years were selected from participants of the Japan Multi-Institutional Collaborative Cohort Study. We documented dietary intakes, measured serum concentrations of oestrone (E1) and oestradiol (E2) and genotyped polymorphisms in oestrogen-metabolising CYP19A1 (rs4441215 and rs936306) and HSD17B1 (rs605059) genes. Trends and interactions were examined using linear regression models. In addition, we calculated the ratios of the oestrogen concentrations of the second to the highest quartiles (Q2-Q4) of dietary intake to those of

Abbreviations: E1, oestrone; E2, oestradiol; HUFA, highly unsaturated fatty acids; SHBG, sex hormone-binding globulin.

* Corresponding author: S. Takagi, fax +81 52744 2971, email sahoko00117@gmail.com 
the lowest quartiles (Q1). After adjustment for potential confounders, E2 was significantly associated with intake of carbohydrate and noodles; ratios of Q4 $v$. Q1 were 1.15 (95\% CI 1.04, 1.28) and 1.15 (95\% CI 1.04, 1.26), respectively. In contrast, E2 levels were inversely associated with intake of total energy, SFA and $n$-3 highly unsaturated fatty acids ( $n$-3 HUFA); ratios of Q4 $v$. Q1 were $0 \cdot 90$ (95\% CI 0.82, $0 \cdot 99$ ), $0 \cdot 89$ ( $95 \%$ CI $0 \cdot 81,0.98)$ and $0.91(95 \% \mathrm{CI} 0.83,1 \cdot 00)$, respectively. In stratified analysis by polymorphisms, the rs 605059 genotype of $H S D 17 B 1$ significantly modified associations of E2 with intake of $n$-3 HUFA and fish; the associations were limited to those with the CC genotype. Macronutrient intakes were associated with serum E2 level, and these associations may be modified by HSD17B1 polymorphism in postmenopausal women.

\section{Key words: Serum oestrogen: Oestradiol: Macronutrients: CYP19A1: HSD17B1}

Breast cancer is the most frequently diagnosed female cancer worldwide, and its incidence rates have been increasing globally, including in Japan, in recent years ${ }^{(1,2)}$. A higher circulating level of oestrogens is an important risk factor for breast cancer $^{(3,4)}$ because oestrogens stimulate the growth of cancer cells in breast tissue ${ }^{(5)}$. After menopause, the production of endogenous oestrogen ceases in the ovaries. However, extragonadal sites such as peripheral adipose tissue continue to synthesise oestrogens through aromatisation of androgens by the cytochrome P450 enzyme ${ }^{(6)}$. The level of oestrogen synthesis in those tissues increases as a function of age and body weight ${ }^{(5)}$. In terms of dietary factors, it has been hypothesised that fat intake increases the risk of postmenopausal breast cancer. However, findings from many studies are inconsistent with each other and an international review by the World Cancer Research Fund/American Institute for Cancer Research concluded that a direct relationship between fat intake and the development of postmenopausal breast cancer is 'limited-suggestive ${ }^{,(7)}$.

Serum oestrogen concentration is a good biomarker for the evaluation of breast cancer risk. Dietary habits may modify breast cancer risk by changing the oestrogen level, and therefore a diet that leads to a lower serum oestrogen level might contribute to reducing the risk of postmenopausal breast cancer. Although many prior studies have reported associations of foods or nutrients with endogenous sex hormones, the findings are inconsistent. Intakes of red meat and dairy products were shown to affect sex hormone levels, whereas total fat or protein was not associated with levels of oestrogens among a Western population $^{(8)}$. Another study reported that higher fat intake was inversely associated with plasma oestradiol (E2) levels ${ }^{(9)}$. However, a meta-analysis of dietary interventions showed that a low-fat diet reduced serum E2 levels ${ }^{(10)}$. In addition to such dietary factors, genetic factors have been suggested to play an important role in the risk of hormone-dependent cancer through the biosynthesis of oestrogens ${ }^{(5,11)}$. Polymorphisms in the CYP19A1 gene that encodes aromatase, an enzyme that converts androgens to oestrogens, were shown to be associated with serum sex hormone levels in Japanese people $\mathrm{e}^{(11-13)}$. The rs605059 polymorphism in the HSD17B1 gene that encodes $17 \beta$-hydroxysteroid dehydrogenase type 1 (17 $\beta$-HSD1), which catalyses the synthesis of E2 from oestrone (E1), has been investigated regarding its association with multiethnic breast cancer risk ${ }^{(14,15)}$.

Currently, information is limited regarding the relationships between macronutrient intakes and blood oestrogen concentrations in postmenopausal Asians. Although higher intakes of total, MUFA and PUFA were shown to be associated with higher E1 levels ${ }^{(16)}$, these findings have not been widely endorsed. Furthermore, as far as we know, no study has shown that polymorphisms of hormone-related genes modify associations between macronutrient intakes and serum oestrogen concentrations. We therefore conducted a cross-sectional study to examine the association of dietary intake, especially intakes of macronutrients, with serum oestrogen levels in postmenopausal Japanese women. At the same time, we tested for effects of interactions between polymorphisms in CYP19A1 and $H S D 17 B 1$ genes and these dietary intakes on the circulating oestrogen concentration.

\section{Methods}

\section{Study subjects}

The Japan Multi-Institutional Collaborative Cohort Study was launched in 2005 to investigate gene-environment interactions in lifestyle-related diseases, especially in cancer. The details of this cohort study have been described elsewhere ${ }^{(17)}$. More than 90000 participants were enroled in the baseline survey from 14 study areas throughout Japan. Participants completed a questionnaire on lifestyles and medical conditions and donated blood samples.

To examine associations of lifestyle, genetic and medical factors by using a subset of this population, 500-600 consecutive subjects were selected in ten study areas and 4519 participants (2124 men and 2395 women) were eventually included in the cross-sectional study ${ }^{(18)}$. Of these subjects, 799 naturally menopaused women aged 47-69 years were selected in nine study areas for the present study ${ }^{(12)}$; one study area was dropped because serum samples were not available for the current study. Natural menopause was defined as no menstruation for at least 12 consecutive months, except for menopause caused by surgeries of the uterus or ovaries or other reasons. We excluded subjects who underwent any hormone therapy when they participated in this study $(n 7)$ and participants whose estimated intakes of total energy were outside the mean ( $\pm 3 \mathrm{sD}$ ) ( $n$ 8). Thus, this analysis was performed in 784 postmenopausal women. This study was conducted according to the guidelines laid down in the Declaration of Helsinki, and all procedures involving human subjects were approved by the ethics committees of Nagoya University Graduate School of Medicine, Aichi Cancer Center and other participating institutions. Written informed consent was obtained from all subjects.

\section{Lifestyle data and serum hormone measurements}

Participants completed a self-administered questionnaire that included questions on anthropometric factors and lifestyles such 
as dietary intakes, alcohol drinking and smoking habits, physical activity and female reproductive factors. Regarding the validity of self-reported height and weight, the Pearson's correlation coefficients between self-reported and actually measured values were 0.96 ( $n$ 268) for height and 0.97 ( $n$ 270) for weight.

For recording dietary intakes, we used a FFQ comprising forty-seven food items ${ }^{(19-21)}$. Regarding staple foods (rice, bread and noodles), subjects were asked about daily intake frequency (six possible categories) and portion size for each of breakfast, lunch and supper. Intake frequencies of other food items, averaged over the previous 1 year, were reported within eight categories. We estimated total energy and macronutrient intakes based on intake frequencies of each food with female standard portion sizes by using the Standard Tables of Food Composition, Version $5^{(22)}$. For alcohol drinking, we asked about drinking habits in the previous 1 year with three potential responses (current drinkers, former drinkers or never drinkers). The amount of alcohol consumption was calculated based on the type of alcoholic beverage (Japanese sake, shochu, shochu highball, beer, whiskey or wine) and the frequency and usual amount of drinking in $1 \mathrm{~d}$ for each beverage. Alcohol consumption was considered energy intake. We also calculated consumption of staple foods and fish; the latter is rich in $n-3$ highly unsaturated fatty acids ( $n-3$ HUFA) that are suggested to be protective against breast cancer ${ }^{(23-25)}$.

The validity of this FFQ was previously evaluated by referring 3 -d weighed diet records in middle-aged Japanese ${ }^{(20)}$. The de-attenuated, log-transformed and energy-adjusted Pearson's correlation coefficients of daily intakes of total energy, protein, fat, carbohydrate, SFA, MUFA, PUFA and $n$-3 HUFA were 0.44 , $0.36,0.48,0.64,0.42,0.34,0.25$ and 0.35 , respectively.

For smoking status, subjects answered whether they smoked with three potential responses (never smokers, former smokers or current smokers). Physical activity was evaluated by computing metabolic equivalent (MET)-h/week from the multiplication of intensity, frequency and times of daily and leisure-time activities. We further asked about female reproductive factors including menstrual status (regular, irregular or ceased), age at menopause and its reason (natural, surgical or others) and history of any hormone therapy.

Serum samples were frozen and stored at $-80^{\circ} \mathrm{C}$ until analysis. Both serum E1 and E2 levels were measured using liquid chromatography-tandem MS (LC-MS/MS), and serum sex hormone-binding globulin (SHBG) was measured using an immunoradiometric assay (IRMA). These analyses were performed at ASKA Pharmaceutical Co., Ltd. The sensitivity of LC-MS/MS was $5.0 \mathrm{pg} / \mathrm{ml}$ for both E1 and E2, and that of IRMA was $0.2 \mathrm{nmol} / 1$ for SHBG. The intra-assay CV were $0 \cdot 4-3 \cdot 3 \%$ for E1, 3.2-6.6\% for E2 and $3.5-6.5 \%$ for SHBG. The inter-assay CV for E1, E2 and SHBG were $3 \cdot 0-3 \cdot 2,4 \cdot 8-9 \cdot 5$ and $1 \cdot 5-8 \cdot 2 \%$, respectively.

\section{SNP selection and genotyping}

To examine the interaction between dietary intakes and genetic factors, we selected two SNP of the CYP19A1 (rs4441215 and rs936306) gene based on our previous report with the same subjects of the present study ${ }^{(12)}$; both E1 and E2 concentrations were associated with these SNP. In addition, the rs605059 polymorphism in the $H S D 17 B 1$ gene was included in the study because several reports have investigated its influence on breast cancer risk ${ }^{(14,15)}$. These selected polymorphisms were genotyped by the multiplex PCR-based Invader assay (Third Wave Technologies) at the Laboratory for Genotyping Development, Center for Genomic Medicine, RIKEN $^{(26)}$.

\section{Statistical analysis}

BMI was calculated based on self-reported height and weight. Values of serum E1 and E2 concentrations were logarithmically transformed for all statistical analyses to approximate to the normal distribution. We excluded subjects whose serum E1 or E2 concentration was ten times or higher than the respective median (E1, $n 1$; E2, $n$ 12). The associations between serum oestrogen levels and potential confounders (age, current BMI, total energy intake, alcohol drinking, physical activity and menopause period) were examined by using Pearson's correlation coefficients. The differences in oestrogen levels among categories of smoking status (never smokers, former smokers, current smokers) and history of hormone therapy use (yes, no) were examined by one-way ANOVA (ANOVA) and $t$ test, respectively.

To examine associations between serum E1 or E2 level and dietary intakes, we used linear regression models regressing serum E1 or E2 concentration on quartiles of each nutrient or food group intake. We used the energy density method to adjust daily intakes of all nutrients and foods for total energy intake (per $4184 \mathrm{~kJ}(1000 \mathrm{kcal}))$. The regression coefficients were adjusted for age at study enrolment (years) and total energy intake $(\mathrm{kJ} / \mathrm{d}$ $(\mathrm{kcal} / \mathrm{d})$ ) in model 1. In model 2, we further adjusted for current BMI $\left(\mathrm{kg} / \mathrm{m}^{2}\right.$ ), smoking status (never smokers, former smokers, current smokers), alcohol drinking (g/d), physical activity (MET$\mathrm{h} /$ week), menopause period (years) and history of any hormone therapy in addition to covariates of model 1 . Tests for trend were performed by assigning medians of nutrient or food group intakes in each quartile. We calculated ratios of serum oestrogen concentrations of the second, third and highest quartiles with 95\% CI to those of the lowest quartile.

In the analysis stratified by genotypes, linear regression analysis for serum oestrogen concentration was performed as in the above-mentioned model 2 . The $P$ values for interactions between dietary intakes and genetic polymorphisms were computed in the linear regression models that included products of the number of minor alleles $(0,1$ or 2$)$ and the median of nutrient or food group intake in each quartile as an independent variable. Genotype frequencies were tested for deviation from the Hardy-Weinberg equilibrium with the genhwi command of Stata version 14 (Stata Corp.). All $P$ values were two-sided and $P$ values $<0.05$ were considered statistically significant. All analyses were performed using Stata version 14.

\section{Results}

The background characteristics, dietary intake and serum oestrogen concentrations of the 784 women are shown in Table 1. The mean age was 60.5 years, and the median total energy intake was estimated as $6406 \mathrm{~kJ} / \mathrm{d}(1531 \mathrm{kcal} / \mathrm{d})$. Table 2 shows 
Table 1. Characteristics, dietary intake and serum oestrogen levels of the subjects $(n 784)$

(Mean values and standard deviations; numbers and percentages; medians and interquartile ranges (IQR))

\begin{tabular}{|c|c|c|}
\hline Variables & Median & IQR \\
\hline \multicolumn{3}{|l|}{ Age (years) } \\
\hline Mean & \multicolumn{2}{|c|}{$60 \cdot 5$} \\
\hline SD & \multicolumn{2}{|c|}{$5 \cdot 1$} \\
\hline \multicolumn{3}{|l|}{ BMI $\left(\mathrm{kg} / \mathrm{m}^{2}\right)$} \\
\hline Mean & \multicolumn{2}{|c|}{$22 \cdot 7$} \\
\hline SD & \multicolumn{2}{|c|}{$3 \cdot 1$} \\
\hline \multicolumn{3}{|l|}{ Physical activity (MET-h/week) } \\
\hline Mean & \multicolumn{2}{|c|}{$14 \cdot 5$} \\
\hline SD & \multicolumn{2}{|c|}{$12 \cdot 5$} \\
\hline \multicolumn{3}{|l|}{ Alcohol drinking } \\
\hline $0 \mathrm{~g} / \mathrm{d}$ & & \\
\hline$n$ & \multicolumn{2}{|c|}{546} \\
\hline$\%$ & \multicolumn{2}{|c|}{$69 \cdot 6$} \\
\hline \multicolumn{3}{|l|}{$<6.0 \mathrm{~g} / \mathrm{d}$} \\
\hline$n$ & \\
\hline$\%$ & \multicolumn{2}{|c|}{$15 \cdot 3$} \\
\hline \multicolumn{3}{|l|}{$\geq 6.0 \mathrm{~g} / \mathrm{d}$} \\
\hline$n$ & \multirow{2}{*}{\multicolumn{2}{|c|}{$\begin{array}{c}118 \\
15 \cdot 1\end{array}$}} \\
\hline$\%$ & & \\
\hline \multicolumn{3}{|l|}{ Smoking status } \\
\hline Never smokers & & \\
\hline$n$ & & \\
\hline$\%$ & & \\
\hline Former smokers & & \\
\hline$n$ & & \\
\hline$\%$ & & \\
\hline Current smokers & & \\
\hline$n$ & & 0 \\
\hline$\%$ & & \\
\hline Menopause period (years)* & & \\
\hline Mean & & 8 \\
\hline SD & & \\
\hline Ever hormone therapy users & & \\
\hline$n$ & & \\
\hline$\%$ & & \\
\hline Dietary intake $(/ d)$ & & \\
\hline Total energy (kJ) & 6418 & $5832-6937$ \\
\hline Total energy (kcal) & 1531 & 1394-1658 \\
\hline Protein $(\mathrm{g})$ & $50 \cdot 1$ & $44 \cdot 3-56 \cdot 7$ \\
\hline Protein (\%E) & $13 \cdot 3$ & $12 \cdot 2-14 \cdot 6$ \\
\hline Fat (g) & 41.9 & $35.5-49.9$ \\
\hline Fat $(\% \mathrm{E})$ & 25.4 & $21 \cdot 9-29.1$ \\
\hline Carbohydrate (g) & 216 & $188-240$ \\
\hline Carbohydrate (\%E) & 61.4 & $56 \cdot 7-65 \cdot 6$ \\
\hline SFA (g) & 11.2 & $9 \cdot 3-12 \cdot 9$ \\
\hline MUFA (g) & $15 \cdot 5$ & $13 \cdot 3-18 \cdot 2$ \\
\hline PUFA (g) & $12 \cdot 7$ & $10 \cdot 9-15 \cdot 5$ \\
\hline$n-3$ PUFA (g) & $2 \cdot 2$ & $1 \cdot 9-2 \cdot 6$ \\
\hline$n-6$ PUFA (g) & 10.5 & $9 \cdot 0-12 \cdot 8$ \\
\hline$n-3$ Highly unsaturated fatty acids (g) & 0.7 & $0.5-1.0$ \\
\hline Cholesterol (mg) & 229 & $183-284$ \\
\hline Rice (g) & 300 & $225-375$ \\
\hline Bread $(g)$ & 35 & $7-70$ \\
\hline Noodles (g) & 25 & $0-38$ \\
\hline Fish $(\mathrm{g})$ & 30 & $12-48$ \\
\hline Serum oestrogen level & & \\
\hline Oestrone (pg/ml) & 14.5 & $10 \cdot 6-19 \cdot 0$ \\
\hline Oestradiol (pg/ml) & 2.55 & $1 \cdot 23-4 \cdot 10$ \\
\hline
\end{tabular}

MET, metabolic equivalent; \%E, percentage of total energy.

* Menopause period was defined as the difference between age at enrolment and age at menopause.

Pearson's correlation coefficients between serum oestrogen levels and background characteristics. BMI was weakly correlated with both serum E1 and E2 concentrations. Age and
Table 2. Correlation coefficients between serum oestrogen levels and characteristics of the subjects

\begin{tabular}{lrrrrr}
\hline & \multicolumn{2}{c}{ Oestrone $(\mathrm{E} 1, n$ 783) } & & \multicolumn{2}{c}{ Oestradiol $(\mathrm{E} 2, n$ 772) } \\
\cline { 2 - 3 } \cline { 6 - 6 } & $r$ & $P$ & & $r$ & $P$ \\
\hline Age & -0.039 & 0.238 & & -0.072 & 0.045 \\
BMI & 0.092 & 0.010 & & 0.101 & 0.005 \\
Total energy intake & -0.010 & 0.774 & & -0.020 & 0.585 \\
Alcohol intake & -0.019 & 0.590 & & -0.010 & 0.779 \\
Physical activity & -0.021 & 0.557 & & 0.035 & 0.334 \\
Menopause period & -0.054 & 0.134 & & -0.080 & 0.026 \\
\hline
\end{tabular}

menopause period were inversely correlated with serum E2, but the correlations were very weak. No significant association was found between either the smoking status or the history of hormone therapy use and serum oestrogens (data not shown).

Tables 3 and 4 summarise the ratios of serum oestrogen concentrations of the second to the highest quartiles (Q2-Q4) of dietary intake to those of the lowest quartile (Q1). None of the macronutrients, including groups of fatty acids, was significantly associated with serum E1 level in models 1 and 2 (Table 3).

Serum E2 concentration was 15\% higher for Q4 of carbohydrate intake than for Q1 in model 2 (ratio: $1 \cdot 15$; $95 \%$ CI 1.04, $1.28 ; P_{\text {trend }}=0.017$ ) (Table 4). For staple foods, noodle intake was positively associated with serum E2 (ratio for Q4 $v$. Q1: $1.15 ; 95 \%$ CI 1.04, 1.26; $P_{\text {trend }}=0.004$ (model 2$)$ ). Only in model 2, Q4 of total energy intake was significantly related to a $10 \%$ lower serum E2 level than Q1 (ratio: 0.90; 95\% CI 0.82, 0.99; $P_{\text {trend }}=0.038$ ). For groups of fatty acids in the model 2 , SFA intake was inversely correlated with serum E2; the ratio of the highest quartile of SFA intake to that of the lowest quartile was 0.89 (95\% CI $\left.0.81,0.98 ; P_{\text {trend }}=0.024\right) . n$-3 HUFA intake was also inversely associated with serum E2 concentration with significance. We further compared the serum E2 level between the quartiles of fish consumption, as fish contains abundant $n-3$ HUFA. The level for Q4 was 9\% lower than that for Q1. However, this association was not significant. No significant correlation of serum E2 level with total protein, total fat or fatty acids except for SFA and $n$-3 HUFA was observed. Furthermore, adjustment for serum SHBG level in model 2 scarcely changed the associations of dietary intakes with serum oestrogen levels (data not shown).

Both the polymorphisms (rs4441215 and rs936306) in the CYP19A1 gene and one polymorphism (rs605059) in the HSD17B1 gene were consistent with the Hardy-Weinberg equilibrium; $P$ values for these polymorphisms were 0.400 , 0.603 and 0.053 , respectively. The minor allele frequencies of rs4441215, rs936306 and rs605059 were 0.37, 0.40 and 0.48, respectively. Table 5 shows assessment of the interaction between each SNP and nutrients or food groups, for dietary factors that were found to have statistically significant or marginally significant associations with serum E2 concentration in the analysis in Table 4. The combination of high carbohydrate intake and the heterozygote (CG) of CYP19A1 rs4441215 showed a higher serum E2 level (ratio of Q4 $v$. Q1: 1.24; $95 \% \mathrm{CI}$ $1 \cdot 06,1.45 ; P_{\text {for trend }}=0 \cdot 015$ ). On the other hand, positive associations between noodle intake and serum E2 level were 
Table 3. Serum oestrone concentration by quartile $(Q)$ of dietary intake $(n 783)$

(Ratios to the lowest quartile and $95 \%$ confidence intervals)

\begin{tabular}{|c|c|c|c|c|c|c|c|c|c|c|c|c|c|c|c|c|}
\hline & \multicolumn{8}{|c|}{ Model $1^{*}$} & \multicolumn{8}{|c|}{ Model $2 \dagger$} \\
\hline & \multirow[b]{2}{*}{ Q1 } & \multicolumn{2}{|r|}{ Q2 } & \multicolumn{2}{|r|}{ Q3 } & \multicolumn{2}{|r|}{ Q4 } & \multirow[b]{2}{*}{$P_{\text {for trend }}$} & \multirow[b]{2}{*}{ Q1 } & \multicolumn{2}{|r|}{ Q2 } & \multicolumn{2}{|r|}{ Q3 } & \multicolumn{2}{|r|}{ Q4 } & \multirow[b]{2}{*}{$P_{\text {for trend }}$} \\
\hline & & Ratio & $95 \% \mathrm{Cl}$ & Ratio & $95 \% \mathrm{Cl}$ & Ratio & $95 \% \mathrm{Cl}$ & & & Ratio & $95 \% \mathrm{Cl}$ & Ratio & $95 \% \mathrm{Cl}$ & Ratio & $95 \% \mathrm{Cl}$ & \\
\hline Total energy & 1.00 & 1.04 & $0.99,1.09$ & 1.03 & $0.98,1.09$ & 1.00 & $0.95,1.05$ & 0.921 & 1.00 & 1.04 & $0.99,1.09$ & 1.04 & $0.99,1.09$ & 1.00 & $0.95,1.06$ & 0.872 \\
\hline Protein & 1.00 & 0.99 & $0.94,1.04$ & 0.96 & $0.91,1.01$ & 0.99 & $0.95,1.05$ & 0.616 & 1.00 & 0.99 & $0.94,1.04$ & 0.96 & $0.91,1.01$ & 1.00 & $0.95,1.05$ & 0.730 \\
\hline Fat & 1.00 & 1.02 & $0.98,1.08$ & 1.03 & $0.98,1.08$ & 1.01 & $0.96,1.07$ & 0.707 & 1.00 & 1.03 & $0.98,1.08$ & 1.03 & $0.98,1.08$ & 1.01 & $0.96,1.07$ & 0.657 \\
\hline Carbohydrate & 1.00 & 1.00 & $0.95,1.05$ & 0.99 & $0.94,1.04$ & 1.04 & $0.98,1.09$ & 0.316 & 1.00 & 0.99 & $0.94,1.05$ & 0.98 & $0.93,1.04$ & 1.03 & $0.97,1.09$ & 0.426 \\
\hline SFA & 1.00 & 1.01 & $0.96,1.06$ & 0.99 & $0.95,1.05$ & 0.97 & $0.92,1.02$ & 0.205 & 1.00 & 1.01 & $0.96,1.06$ & 0.99 & $0.94,1.04$ & 0.98 & $0.93,1.03$ & 0.263 \\
\hline MUFA & 1.00 & 1.03 & $0.98,1.08$ & 1.00 & $0.95,1.05$ & 1.02 & $0.97,1.08$ & 0.643 & 1.00 & 1.03 & $0.98,1.08$ & 1.00 & $0.95,1.05$ & 1.02 & $0.97,1.08$ & 0.659 \\
\hline PUFA & 1.00 & 1.02 & $0.97,1.07$ & 1.04 & $0.98,1.09$ & 1.03 & $0.98,1.08$ & 0.297 & 1.00 & 1.02 & $0.97,1.07$ & 1.03 & $0.98,1.09$ & 1.03 & $0.97,1.08$ & 0.325 \\
\hline Cholesterol & 1.00 & 0.95 & $0.91,1.00$ & 1.04 & $0.99,1.09$ & 1.01 & $0.97,1.07$ & 0.175 & 1.00 & 0.95 & $0.91,1.00$ & 1.04 & $0.99,1.09$ & 1.02 & $0.97,1.07$ & 0.111 \\
\hline$n-3$ PUFA & 1.00 & 0.97 & $0.93,1.02$ & 0.97 & $0.93,1.02$ & 1.00 & $0.95,1.05$ & 0.888 & 1.00 & 0.97 & $0.93,1.02$ & 0.97 & $0.93,1.02$ & 1.00 & $0.95,1.05$ & 0.997 \\
\hline$n-6$ PUFA & 1.00 & 0.99 & $0.94,1.04$ & 1.01 & $0.96,1.06$ & 1.02 & $0.97,1.07$ & 0.362 & 1.00 & 0.99 & $0.94,1.04$ & 1.01 & $0.96,1.06$ & 1.02 & $0.96,1.07$ & 0.399 \\
\hline$n-3$ Highly unsaturated fatty acids & 1.00 & 0.97 & $0.92,1.02$ & 0.99 & $0.94,1.04$ & 0.97 & $0.92,1.02$ & 0.350 & 1.00 & 0.97 & $0.92,1.02$ & 0.99 & $0.94,1.04$ & 0.97 & $0.93,1.03$ & 0.446 \\
\hline Rice & 1.00 & 1.02 & $0.97,1.07$ & 1.01 & $0.96,1.06$ & 1.01 & $0.96,1.07$ & 0.545 & 1.00 & 1.02 & $0.97,1.08$ & 1.01 & $0.96,1.07$ & 1.01 & $0.96,1.07$ & 0.744 \\
\hline Bread & 1.00 & 1.01 & $0.96,1.06$ & 1.04 & $0.99,1.09$ & 0.99 & $0.94,1.04$ & 0.980 & 1.00 & 1.01 & $0.96,1.06$ & 1.04 & $0.99,1 \cdot 10$ & 0.99 & $0.94,1.05$ & 0.899 \\
\hline Noodles & 1.00 & 1.03 & $0.98,1.08$ & 1.04 & $0.99,1.09$ & 1.02 & $0.97,1.07$ & 0.578 & 1.00 & 1.03 & $0.97,1.08$ & 1.04 & $0.99,1.09$ & 1.02 & $0.97,1.07$ & 0.542 \\
\hline Fish & 1.00 & 0.98 & $0.93,1.03$ & 0.96 & $0.91,1.01$ & 0.99 & $0.94,1.04$ & 0.646 & 1.00 & 0.98 & $0.93,1.03$ & 0.96 & $0.92,1.01$ & 0.99 & $0.95,1.05$ & 0.811 \\
\hline
\end{tabular}

* Model 1: adjusted for age and total energy intake.

† Model 2: adjusted for age, current BMI, smoking status, alcohol drinking, physical activity, total energy intake, menopause period and history of any hormone therapy.

Table 4. Serum oestradiol concentration by quartile $(\mathrm{Q})$ of dietary intake $(n 772)$

(Ratios to the lowest quartile and $95 \%$ confidence intervals)

\begin{tabular}{|c|c|c|c|c|c|c|c|c|c|c|c|c|c|c|c|c|}
\hline & \multicolumn{8}{|c|}{ Model $1^{*}$} & \multicolumn{8}{|c|}{ Model $2 \dagger$} \\
\hline & \multirow[b]{2}{*}{ Q1 } & \multicolumn{2}{|r|}{ Q2 } & \multicolumn{2}{|r|}{ Q3 } & \multicolumn{2}{|r|}{ Q4 } & \multirow[b]{2}{*}{$P_{\text {for trend }}$} & \multirow[b]{2}{*}{ Q1 } & \multicolumn{2}{|r|}{ Q2 } & \multicolumn{2}{|r|}{ Q3 } & \multicolumn{2}{|r|}{ Q4 } & \multirow[b]{2}{*}{$P_{\text {for trend }}$} \\
\hline & & Ratio & $95 \% \mathrm{Cl}$ & Ratio & $95 \% \mathrm{Cl}$ & Ratio & $95 \% \mathrm{Cl}$ & & & Ratio & $95 \% \mathrm{Cl}$ & Ratio & $95 \% \mathrm{Cl}$ & Ratio & $95 \% \mathrm{Cl}$ & \\
\hline Total energy & 1.00 & 0.98 & $0.89,1.08$ & 0.98 & $0.89,1.08$ & 0.91 & $0.83,1.00$ & 0.058 & 1.00 & 0.98 & $0.89,1.07$ & 0.97 & $0.89,1.07$ & 0.90 & $0.82,0.99$ & 0.038 \\
\hline Protein & 1.00 & 0.93 & $0.85,1.02$ & 0.92 & $0.84,1.01$ & 0.92 & $0.84,1.02$ & 0.123 & 1.00 & 0.94 & $0.85,1.03$ & 0.93 & $0.85,1.02$ & 0.93 & $0.84,1.02$ & 0.134 \\
\hline Fat & 1.00 & 1.02 & $0.93,1.12$ & 0.97 & $0.88,1.07$ & 0.99 & $0.90,1.09$ & 0.594 & 1.00 & 1.03 & $0.93,1.13$ & 0.98 & $0.89,1.08$ & 0.99 & $0.90,1.09$ & 0.646 \\
\hline Carbohydrate & 1.00 & $1 \cdot 10$ & $1.01,1.21$ & 1.06 & $0.96,1.17$ & $1 \cdot 15$ & $1.04,1.27$ & 0.015 & 1.00 & $1 \cdot 11$ & $1.00,1.23$ & 1.07 & $0.97,1.19$ & $1 \cdot 15$ & $1.04,1.28$ & 0.017 \\
\hline SFA & 1.00 & 0.95 & $0.87,1.05$ & 0.96 & $0.87,1.06$ & 0.89 & $0.80,0.98$ & 0.020 & 1.00 & 0.96 & $0.87,1.05$ & 0.96 & $0.87,1.05$ & 0.89 & $0.81,0.98$ & 0.024 \\
\hline MUFA & 1.00 & 1.06 & $0.97,1.17$ & 0.99 & $0.90,1.09$ & 1.05 & $0.95,1.16$ & 0.593 & 1.00 & 1.06 & $0.96,1.16$ & 0.99 & $0.90,1.09$ & 1.04 & $0.94,1.15$ & 0.704 \\
\hline PUFA & 1.00 & 1.06 & $0.96,1.17$ & 1.02 & $0.93,1.13$ & 1.02 & $0.93,1.13$ & 0.933 & 1.00 & 1.06 & $0.96,1.16$ & 1.02 & $0.93,1.12$ & 1.01 & $0.92,1.12$ & 0.920 \\
\hline Cholesterol & 1.00 & 0.91 & $0.83,1.00$ & 0.96 & $0.87,1.05$ & 0.96 & $0.87,1.05$ & 0.674 & 1.00 & 0.91 & $0.83,1.00$ & 0.96 & $0.87,1.06$ & 0.97 & $0.88,1.06$ & 0.829 \\
\hline$n-3$ PUFA & 1.00 & 1.01 & $0.91,1.11$ & 0.98 & $0.89,1.07$ & 0.99 & $0.89,1.09$ & 0.721 & 1.00 & 1.01 & $0.92,1.11$ & 0.98 & $0.89,1.08$ & 0.98 & $0.89,1.08$ & 0.562 \\
\hline$n-6$ PUFA & 1.00 & 1.01 & $0.92,1 \cdot 11$ & 1.04 & $0.94,1.14$ & 1.03 & $0.94,1.14$ & 0.461 & 1.00 & 1.01 & $0.92,1.11$ & 1.04 & $0.94,1.14$ & 1.02 & $0.93,1.13$ & 0.596 \\
\hline$n-3$ Highly unsaturated fatty acids & 1.00 & 1.00 & $0.91,1 \cdot 11$ & 0.98 & $0.89,1.08$ & 0.91 & $0.82,1.00$ & 0.031 & 1.00 & 1.00 & $0.91,1 \cdot 11$ & 0.99 & $0.89,1.08$ & 0.91 & $0.83,1.00$ & 0.042 \\
\hline Rice & 1.00 & $1 \cdot 10$ & $1.01,1.21$ & 1.09 & $0.99,1.19$ & 1.05 & $0.94,1.14$ & 0.171 & 1.00 & $1 \cdot 13$ & $1.03,1.25$ & $1 \cdot 11$ & $1.01,1.23$ & 1.07 & $0.96,1 \cdot 19$ & 0.267 \\
\hline Bread & 1.00 & 1.17 & $1.07,1.29$ & $1 \cdot 10$ & $1.00,1.21$ & 1.04 & $0.94,1.14$ & 0.973 & 1.00 & $1 \cdot 17$ & $1.07,1.29$ & $1 \cdot 12$ & $1.02,1.23$ & 1.05 & $0.96,1.16$ & 0.653 \\
\hline Noodles & 1.00 & 1.06 & $0.97,1.17$ & $1 \cdot 18$ & $1.07,1.29$ & $1 \cdot 14$ & $1.04,1.25$ & 0.007 & 1.00 & 1.06 & $0.96,1.17$ & $1 \cdot 18$ & $1 \cdot 08,1 \cdot 30$ & $1 \cdot 15$ & $1 \cdot 04,1 \cdot 26$ & 0.004 \\
\hline Fish & 1.00 & 1.00 & $0.91,1 \cdot 10$ & 1.04 & $0.94,1.14$ & 0.91 & $0.82,1.00$ & 0.043 & 1.00 & 1.00 & $0.91,1 \cdot 10$ & 1.04 & $0.95,1.14$ & 0.91 & $0.83,1.01$ & 0.066 \\
\hline
\end{tabular}

Model 1: adjusted for age and total energy intake.

† Model 2: adjusted for age, current BMI, smoking status, alcohol drinking, physical activity, total energy intake, menopause period and history of any hormone therapy. 
Writish Journal of Nutrition

Table 5. Serum oestradiol concentration by quartile of dietary intake: analysis stratified by CYP19A1 rs4441215 and rs936306 and HSD17B1 rs605059 genotypes ( $n$ 772) ${ }^{\star}$ (Ratios to the lowest quartile and $95 \%$ confidence intervals)

\begin{tabular}{|c|c|c|c|c|c|c|c|c|c|c|c|}
\hline \multirow[b]{2}{*}{ Polymorphism } & & \multirow[b]{2}{*}{ Dietary intakes } & \multirow[b]{2}{*}{ Genotype (n) } & \multirow[b]{2}{*}{ Q1 } & \multicolumn{2}{|c|}{ Q2 } & \multicolumn{2}{|c|}{ Q3 } & \multicolumn{2}{|c|}{ Q4 } & \multirow[b]{2}{*}{$P_{\text {for trend }}$} \\
\hline & & & & & Ratio & $95 \% \mathrm{Cl}$ & Ratio & $95 \% \mathrm{Cl}$ & Ratio & $95 \% \mathrm{Cl}$ & \\
\hline \multirow[t]{24}{*}{ CYP19A1 } & rs 4441215 & Total energy & CC (311) & 1.00 & 1.00 & $0.87,1.15$ & 1.02 & $0.88,1 \cdot 19$ & 0.90 & $0.78,1.04$ & 0.204 \\
\hline & & & CG (350) & 1.00 & 0.95 & $0.82,1 \cdot 10$ & 1.01 & $0.87,1.16$ & 0.87 & $0.76,1.00$ & 0.095 \\
\hline & & & GG (111) & 1.00 & $1 \cdot 10$ & $0.81,1.49$ & 0.89 & $0.65,1.21$ & 1.07 & $0.79,1.45$ & 0.865 \\
\hline & & & & & & & $P_{\text {for interaction }}=$ & & & & \\
\hline & & Carbohydrate & $\mathrm{CC}$ & 1.00 & $1 \cdot 18$ & $1.02,1.38$ & 1.08 & $0.93,1.27$ & 1.07 & $0.91,1.25$ & 0.543 \\
\hline & & & CG & 1.00 & 1.15 & $0.99,1.33$ & $1 \cdot 10$ & $0.94,1.28$ & $1 \cdot 24$ & $1.06,1.45$ & 0.015 \\
\hline & & & $\mathrm{GG}$ & 1.00 & 0.84 & $0.62,1.14$ & 1.01 & $0.75,1.35$ & 1.13 & $0.82,1.56$ & 0.385 \\
\hline & & & & & & & $P_{\text {for interaction }}=$ & & & & \\
\hline & & SFA & $\mathrm{CC}$ & 1.00 & 0.95 & $0.82,1 \cdot 10$ & 1.04 & $0.90,1.20$ & 0.90 & $0.77,1.04$ & 0.263 \\
\hline & & & $C G$ & 1.00 & 0.99 & $0.86,1 \cdot 14$ & 0.93 & $0.80,1.07$ & 0.91 & $0.77,1.04$ & 0.095 \\
\hline & & & $\mathrm{GG}$ & 1.00 & 0.95 & $0.71,1.27$ & 0.83 & $0.60,1 \cdot 14$ & 0.91 & $0.68,1.21$ & 0.480 \\
\hline & & & & & & & $P_{\text {for interaction }}=$ & & & & \\
\hline & & $n-3$ HUFA & CC & 1.00 & 0.89 & $0.77,1.04$ & 0.99 & $0.85,1.14$ & 0.83 & $0.72,0.95$ & 0.019 \\
\hline & & & CG & 1.00 & 1.11 & $0.96,1.28$ & 1.00 & $0.87,1.15$ & 1.01 & $0.87,1.17$ & 0.825 \\
\hline & & & $\mathrm{GG}$ & 1.00 & 0.96 & $0.71,1.30$ & 1.53 & $0.67,1.29$ & 0.84 & $0.62,1.15$ & 0.265 \\
\hline & & & & & & & $P_{\text {for interaction }}=$ & & & & \\
\hline & & Noodles & $\mathrm{CC}$ & 1.00 & 1.06 & $0.91,1 \cdot 22$ & 1.21 & $1.05,1.40$ & $1 \cdot 16$ & $1 \cdot 01,1 \cdot 34$ & 0.041 \\
\hline & & & CG & 1.00 & 1.05 & $0.91,1.21$ & $1 \cdot 10$ & $0.96,1.26$ & 1.08 & $0.94,1.25$ & 0.317 \\
\hline & & & $\mathrm{GG}$ & 1.00 & 1.25 & $0.95,1.63$ & 1.53 & $1.13,2.06$ & 1.39 & $1.03,1.88$ & 0.032 \\
\hline & & & & & & & $P_{\text {for interaction }}=$ & & & & \\
\hline & & Fish & $\mathrm{CC}$ & 1.00 & 0.92 & $0.79,1.06$ & 0.99 & $0.86,1.14$ & 0.83 & $0.72,0.96$ & 0.015 \\
\hline & & & $C G$ & 1.00 & 1.08 & $0.93,1.24$ & $1 \cdot 10$ & $0.95,1.26$ & 1.01 & $0.88,1.17$ & 0.857 \\
\hline & & & $\mathrm{GG}$ & 1.00 & 1.00 & $0.74,1.35$ & 1.03 & $0.75,1.40$ & 0.84 & $0.62,1.14$ & 0.202 \\
\hline & & & & & & & $P_{\text {for interaction }}=$ & & & & \\
\hline \multirow[t]{12}{*}{ CYP19A1 } & rs936306 & Total energy & CC (281) & 1.00 & 0.98 & $0.83,1 \cdot 16$ & 0.95 & $0.80,1 \cdot 13$ & 0.87 & $0.74,1.02$ & 0.087 \\
\hline & & & CT (362) & 1.00 & 1.01 & $0.87,1 \cdot 16$ & 1.00 & $0.86,1 \cdot 15$ & 0.93 & $0.81,1.08$ & 0.335 \\
\hline & & & TT (129) & 1.00 & 0.87 & $0.70,1.08$ & 1.01 & $0.81,1.25$ & 0.92 & $0.73,1.14$ & 0.615 \\
\hline & & & & & & & $P_{\text {for interaction }}=$ & & & & \\
\hline & & Carbohydrate & $\mathrm{CC}$ & 1.00 & $1 \cdot 10$ & $0.93,1.31$ & 1.09 & $0.92,1.30$ & $1 \cdot 18$ & $0.98,1.42$ & 0.093 \\
\hline & & & $\mathrm{CT}$ & 1.00 & $1 \cdot 10$ & $0.95,1.29$ & 1.07 & $0.91,1.25$ & 1.13 & $0.96,1.32$ & 0.183 \\
\hline & & & TT & 1.00 & 1.15 & $0.93,1.42$ & 1.06 & $0.86,1.32$ & 1.19 & $0.95,1.50$ & 0.173 \\
\hline & & & & & & & $P_{\text {for interaction }}=$ & & & & \\
\hline & & SFA & $\mathrm{CC}$ & 1.00 & 0.86 & $0.73,1.01$ & 0.94 & $0.80,1.12$ & 0.84 & $0.70,0.99$ & 0.094 \\
\hline & & & CT & 1.00 & 0.99 & $0.86,1 \cdot 13$ & 0.91 & $0.79,1.04$ & 0.91 & $0.79,1.06$ & 0.146 \\
\hline & & & $\mathrm{TT}$ & 1.00 & 1.11 & $0.88,1.39$ & 1.05 & $0.84,1.30$ & 0.95 & $0.75,1 \cdot 19$ & 0.405 \\
\hline & & & & & & & $P_{\text {for interaction }}=$ & & & & \\
\hline \multirow[t]{12}{*}{ CYP19A1 } & rs936306 & $n-3$ HUFA & $\mathrm{CC}$ & 1.00 & 1.01 & $0.85,1.20$ & 0.99 & $0.83,1.17$ & 0.92 & $0.78,1.08$ & 0.247 \\
\hline & & & СT & 1.00 & 1.02 & $0.88,1 \cdot 18$ & 1.00 & $0.87,1 \cdot 16$ & 0.93 & $0.80,1.09$ & 0.291 \\
\hline & & & $\mathrm{TT}$ & 1.00 & 0.98 & $0.79,1.21$ & 0.93 & $0.76,1.13$ & 0.93 & $0.74,1.17$ & 0.452 \\
\hline & & & & & & & $P_{\text {for interaction }}=$ & & & & \\
\hline & & Noodles & $\mathrm{CC}$ & 1.00 & $1 \cdot 16$ & $0.99,1.35$ & 1.21 & $1.03,1.43$ & $1 \cdot 19$ & $1.01,1.40$ & 0.077 \\
\hline & & & CT & 1.00 & 1.06 & $0.91,1.23$ & $1 \cdot 18$ & $1.03,1.35$ & $1 \cdot 14$ & $0.99,1.31$ & 0.087 \\
\hline & & & TT & 1.00 & 0.87 & $0.71,1.08$ & $1 \cdot 17$ & $0.94,1.46$ & 1.07 & $0.88,1.31$ & 0.226 \\
\hline & & & & & & & $P_{\text {for interaction }}=$ & & & & \\
\hline & & Fish & $\mathrm{CC}$ & 1.00 & 1.02 & $0.86,1.20$ & 1.01 & $0.85,1 \cdot 19$ & 0.93 & $0.79,1.09$ & 0.391 \\
\hline & & & CT & 1.00 & 1.02 & $0.88,1 \cdot 18$ & 1.11 & $0.96,1.28$ & 0.95 & $0.82,1.11$ & 0.537 \\
\hline & & & TT & 1.00 & 0.98 & $0.81,1.20$ & 0.95 & $0.76,1.18$ & 0.87 & $0.70,1.07$ & 0.158 \\
\hline & & & & & & & $P_{\text {for interaction }}=$ & & & & \\
\hline \multirow[t]{8}{*}{$H S D 17 B 1$} & rs605059 & Total energy & CC (226) & 1.00 & 0.84 & $0.71,0.99$ & 0.90 & $0.76,1.07$ & 0.85 & $0.71,1.02$ & 0.119 \\
\hline & & & CT (359) & 1.00 & 1.07 & $0.92,1.25$ & 1.02 & $0.87,1.18$ & 0.93 & $0.80,1.08$ & 0.244 \\
\hline & & & ТT (187) & 1.00 & 0.99 & $0.82,1.19$ & 1.00 & $0.82,1.22$ & 0.89 & $0.75,1.07$ & 0.244 \\
\hline & & & & & & & $P_{\text {for interaction }}=$ & & & & \\
\hline & & Carbohydrate & $\mathrm{CC}$ & 1.00 & 1.00 & $0.84,1 \cdot 18$ & 1.20 & $1.00,1.43$ & 1.24 & $1.03,1.48$ & 0.009 \\
\hline & & & CT & 1.00 & 1.19 & $1.01,1.40$ & 1.03 & $0.88,1.21$ & $1 \cdot 15$ & $0.98,1.36$ & 0.270 \\
\hline & & & TT & 1.00 & 1.03 & $0.85,1.25$ & 1.00 & $0.81,1.24$ & 1.02 & $0.82,1.25$ & 0.930 \\
\hline & & & & & & & $P_{\text {for interaction }}=$ & & & & \\
\hline
\end{tabular}




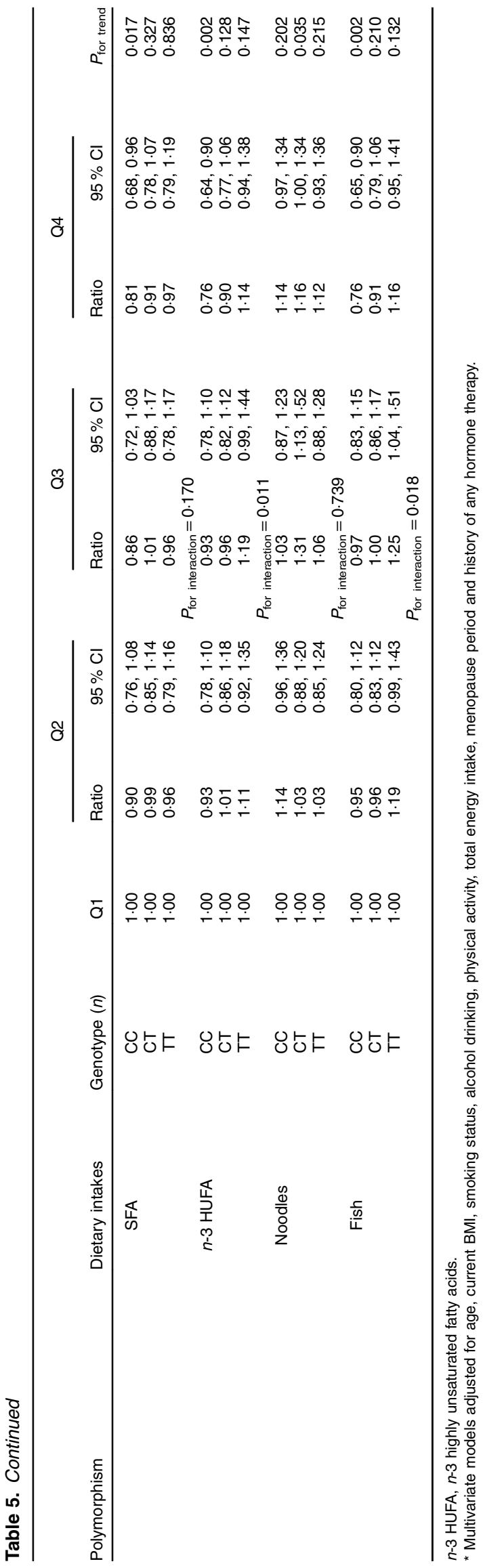

observed in the homozygotes (CC or GG). In addition, the highest quartile of fish consumption with the CC genotype was linked to $17 \%$ lower serum E2 than the lowest quartile (ratio: $0.83 ; 95 \%$ CI $0.72,0.96 ; P_{\text {for trend }}=0.015$ ). However, the interactions of all these associations for this SNP were not statistically significant. Stratification by the rs936306 genotype in CYP19A1 showed neither significant associations nor interactions for serum E2 concentrations. Among women who carried the CC genotype of rs605059 in the HSD17B1 gene, carbohydrate intake was positively associated with serum E2 level (ratio of Q4 v. Q1: 1.24; $95 \%$ CI 1.03, 1.48). On the other hand, intakes of $n-3$ HUFA and fish were inversely associated with serum E2; E2 concentrations of the highest quartile of both $n-3$ HUFA and fish were $24 \%$ lower than those for the lowest quartile. We found statistically significant, or marginally significant, interactions between this SNP and these three nutrients or foods for serum E2 concentration $\left(P_{\text {for interaction }}<0 \cdot 05\right)$. Although the interaction was not significant, SFA intake was also inversely associated with serum E2 in women with the CC genotype (ratio of Q4 $v$. Q1: 0.81; 95\% CI 0.68, 0.96). We found no substantial modification in the association between total energy intake and serum E2 level by any of the three polymorphisms.

\section{Discussion}

In this cross-sectional study of postmenopausal Japanese women, serum E2 concentration was significantly associated with intakes of carbohydrate, especially of noodles. In contrast, the E2 level was inversely correlated with intakes of total energy, SFA and $n$-3 HUFA. In stratified analyses by genetic polymorphisms, we found significant interactions between the rs605059 genotype of the HSD17B1 gene and intakes of $n-3$ HUFA and fish; the associations of serum E2 concentration with these nutrients or foods were limited to those with the CC genotype.

We found statistically significant but weak associations between dietary intakes and serum E2 concentrations in apparently healthy postmenopausal women; compared with the lowest quartiles of intakes of carbohydrate and n-3 HUFA, serum E2 levels were only 15\% higher and 9\% lower in the highest quartiles of the respective nutrient intake. However, in three studies on associations of endogenous sex hormone levels with breast cancer risk in postmenopausal women, in which serum E2 was measured by using MS, the median levels of serum E2 were only $1 \cdot 5-15 \cdot 6 \%$ higher in the breast cancer patients than in the controls ${ }^{(27-29)}$. All three of those studies concluded that higher serum E2 was significantly associated with an increase in the risk of postmenopausal breast cancer ${ }^{(30)}$. We therefore consider that even small differences in E2 levels observed in the present study may be relevant to the health of postmenopausal women.

Although serum oestrogen concentrations in our population were relatively low, they are similar to the oestrogen concentrations of postmenopausal Japanese women reported in another study in which serum oestrogen was measured by using the same method ${ }^{(31)}$. In addition, median serum values were 14.5 (interdecile ranges $7 \cdot 0-24 \cdot 1$ ) $\mathrm{pg} / \mathrm{ml}$ for E1 and 2.55 (interdecile ranges $0.4-5.4) \mathrm{pg} / \mathrm{ml}$ for E2 in our study; 90th 
percentiles were more than 3 and 13 times higher than 10th percentiles for E1 and E2, respectively. These ranges of serum oestrogen levels were comparable with those in previous studies of postmenopausal women that used $\mathrm{MS}^{(27-29)}$. Although we cannot conclude that the measurement of serum oestrogen levels using MS resolves the previous contradictory findings, the current study provided sufficient dynamic range in serum oestrogen concentrations.

Our finding of an inverse association between total energy intake and serum E2 concentration is not in line with previous reports. In two weight-loss intervention studies among postmenopausal women, restriction of dietary energy reduced serum $\mathrm{E} 2^{(32,33)}$. In particular, combinations of dietary energy restriction and exercise had stronger effects on serum sex hormone levels through greater weight loss than dietary interventions alone $^{(32)}$. The discordances between these results and the results of our study may arise from differences between interventional and cross-sectional studies.

As mentioned above, we observed positive and significant associations of the serum E2 concentration and intake of carbohydrate, especially of noodles. A previous study reported that substitution of fat intake for carbohydrate intake of equivalent energy reduced plasma E2 levels ${ }^{(9)}$. Previous results from some prospective cohort studies that were focused on postmenopausal breast cancer risk were inconsistent; higher carbohydrate intake tended to increase overall breast cancer risk, but this association was limited to oestrogen receptor (ER) - or ER-/progesterone receptor (PR) - breast cancer that is independent of endogenous hormone concentrations ${ }^{(34,35)}$. As far as we know, direct associations between noodle intake and serum sex hormone concentration or breast cancer have never been reported. Elderly Japanese people with dietary patterns characterised by higher noodle intake have been reported to tend to consume more processed meat, non-alcoholic soft drinks and beer ${ }^{(36)}$. However, this tendency was not reproduced in our population.

Dietary SFA were inversely associated with serum E2 in our study, whereas two other studies have reported no association among postmenopausal women ${ }^{(8,37)}$. No effects of SFA intake on breast cancer risk were shown in an international pooled analysis $^{(38)}$. It is unlikely that SFA increases breast cancer risk mediated by endogenous oestrogen. Intake of SFA was derived mostly from chicken eggs, whole milk, well-milled rice, pork and beef in the Japanese diet ${ }^{(39)}$. No association was reported between serum E2 levels and intakes of eggs or red meat, whereas a positive association was found with dairy products among a European population ${ }^{(8)}$. These disagreements may arise from the difference in SFA intake and sources between Japanese and Western people.

We found that the serum E2 level was reduced among women with a higher intake of $n-3$ HUFA. Our findings are inconsistent with the results of another study that showed a null association of serum oestrogen concentration with fish consumption $^{(8)}$. However, a different study reported an inverse association between intake of $n-3$ fatty acids from fish and E2 levels ${ }^{(9)}$. Furthermore, our results may be consistent with the finding that higher intakes of $n-3$ HUFA or fish fat tend to decrease breast cancer risk, and that high erythrocyte compositions of these fatty acids were inversely correlated with this risk among Japanese women ${ }^{(23,24)}$. A meta-analysis also indicated that higher intake of marine $n-3$ PUFA was associated with decreased risk of breast cancer and that this association was stronger in Asia than in other areas ${ }^{(25)}$. Plasma concentrations of total $n-3$ HUFA, EPA and DHA, which are derived from marine foods, were moderately and positively correlated with intake of fatty acids ${ }^{(40,41)}$. These fatty acids, especially EPA, would alter oestrogen metabolism. PGE2, which is an inflammatory eicosanoid that is synthesised from arachidonic acid (AA), has been shown to stimulate the expression and activity of aromatase $\mathrm{P} 450$ that converts androgens to oestrogens. In contrast, PGE3 that is derived from EPA does not affect aromatase $\mathrm{P} 450$ activity and has been predicted to inhibit synthesis of PGE2 from $\mathrm{AA}^{(42-44)}$. Therefore, higher intake of EPA may lead to an increase in PGE3 production and a decline in PGE2 production, and thereby a decrease in oestrogenstimulated cell growth through a reduction in oestrogen synthesis.

In the analyses for interaction between dietary intake and genotypes of oestrogen-synthesis-related genes, we focused on the CYP19A1 and HSD17B1 genes. The CYP19A1 gene encodes aromatase, which is a cytochrome P450 enzyme that converts androgens to oestrogens ${ }^{(11)}$. A previous study of the same population of our investigation reported that both serum $\mathrm{E} 1$ and $\mathrm{E} 2$ concentrations were higher in those with the $\mathrm{C}$ allele of rs4441215 or the T allele of rs936306 of the CYP19A1 gene than postmenopausal women without these alleles ${ }^{(12)}$. To our knowledge, this is the first study to explore interactions between intakes of nutrients or foods and these polymorphisms. However, no significant associations were observed.

The HSD17B1 gene is located on chromosome 17q12-q21, and it produces $17 \beta$-HSD1 that increases oestrogen bioactivity by converting E1 to E2. The rs605059 polymorphism of this gene leads to an amino acid change from serine ( $\mathrm{T}$ allele) to glycine (C allele) at position 312, although this change alters neither the catalytic nor the immunological properties of the enzyme ${ }^{(45)}$. According to the SNP database of the National Center for Biotechnology Information, the allele frequency of this polymorphism varies between races; the $\mathrm{C}$ allele is the major allele among Asians, whereas the $\mathrm{T}$ allele is dominant among Europeans ${ }^{(46)}$. Previous studies of this polymorphism with reference to breast cancer risk or blood sex hormone concentrations yielded mixed results. In Asia, postmenopausal Chinese women who carried the AA (or TT) genotype were at an increased risk of breast cancer ${ }^{(47)}$. However, there has been no study that indicated any associations among Japanese women ${ }^{(15,48)}$. In America, higher E2 levels were observed among postmenopausal lean women with the AA (or TT) genotype, whereas no overall effect of the genotype was reported on breast cancer risk ${ }^{(49)}$. Furthermore, two meta-analyses of multiethnic subjects showed no association of rs605059 with breast cancer risk ${ }^{(14,50)}$. To our knowledge, we are the first to explore the interaction between macronutrient intake and the HSD17B1 rs605059 polymorphism with serum sex hormone concentrations. Given the established evidence of the positive association between serum E2 and postmenopausal breast cancer risk ${ }^{(3,4)}$, we suggest that breast cancer risk among postmenopausal women with the CC genotype may be decreased by taking less carbohydrate and more $n-3$ HUFA or fish. Further 
research may elucidate the interaction between genetic background and dietary factors for the effect on serum oestrogen level and breast cancer risk.

The main strength of our study was that serum oestrogen concentrations were measured by using LC-MS/MS. This method detects oestrogens with higher sensitivity than other methods usually used to measure blood sex hormone concentrations of postmenopausal women, which meant that we could exactly assess very low serum oestrogen concentrations in postmenopausal women. In addition, we could consider many potential confounders that have been suggested to affect sex hormone concentrations and examine interactions between dietary factors and genetic polymorphisms.

Several limitations of this study also need to be mentioned. First, we estimated dietary intakes by using a FFQ with moderate validity ${ }^{(20)}$. Although the validity of FFQ in the present study is comparable to that in other large prospective cohort studies in Japan ${ }^{(51,52)}$, an error in dietary assessment is likely to lead to a non-differential misclassification and to attenuate associations between dietary intakes and serum oestrogen levels. Second, we could not clearly show cause-effect relationships owing to the nature of a cross-sectional study. Indeed, a previous study showed that serum levels of oestrogens were positively associated with leptin, which influences appetite regardless of obesity status, and that low-level oestrogens may influence feeding behaviour in postmenopausal women ${ }^{(53)}$. Therefore, further longitudinal or intervention studies are required to reveal causal relationships.

In conclusion, we found significant and positive associations between serum E2 levels and intakes of carbohydrate or noodles, whereas inverse correlations were observed between E2 levels and intakes of total energy, saturated fatty acids or $n-3$ HUFA among postmenopausal Japanese women. A polymorphism of $H S D 17 B 1$, which encodes one of the genes involved in oestrogen synthesis, modified the relationship between dietary intakes and circulating E2 levels. These findings are of significance in terms of discussing the role of diet in the risk of hormone-dependent cancer.

\section{Acknowledgements}

The authors thank Shinkan Tokudome at the National Institute of Health and Nutrition (formerly Nagoya City University); Chiho Goto at Nagoya Bunri University; Nahomi Imaeda at Shigakkan University; Yuko Tokudome at Nagoya University of Arts and Sciences; Masato Ikeda at the University of Occupational and Environmental Health; Shinzo Maki at the Aichi Prefectural Dietetic Association for providing the FFQ and programmes for calculation of dietary intakes; the assistant staff at the Laboratory for Genotyping Development, Center for Genomic Medicine, RIKEN for support with genotyping; and all of the participants and staff who took part in the Japan MultiInstitutional Collaborative Cohort Study for cooperation.

This study was supported by Grants-in-Aid for Scientific Research for Priority Areas of Cancer (no. 17015018) and Innovative Areas (no. 221S0001) and by JSPS KAKENHI Grants (nos 16H06277 and 15H02524) from the Japanese Ministry of Education, Culture, Sports, Science and Technology.
S. T., S. H. and K. W.: formulating the research questions; S. T., C. N., S. H. and K. W.: designing the study; Y. N., N. K., S. S., I. S., H. M., S. H., M. N., S. K., R. O., H. U., N. K., M. K., N. H. and H. T.: carrying out the study; S. T. and K. W.: analysing the data; S. T., C. N., H. U., H. T., K. W.: writing the article.

The authors declare that there are no conflicts of interest.

\section{References}

1. Torre LA, Siegel RL, Ward EM, et al. (2016) Global cancer incidence and mortality rates and trends - an update. Cancer Epidemiol Biomarkers Prev 25, 16-27.

2. Katanoda K, Matsuda T, Matsuda A, et al. (2013) An updated report of the trends in cancer incidence and mortality in Japan. Jpn J Clin Oncol 43, 492-507.

3. Key T, Appleby P, Barnes I, et al. (2002) Endogenous sex hormones and breast cancer in postmenopausal women: reanalysis of nine prospective studies. J Natl Cancer Inst 94, 606-616.

4. Missmer SA, Eliassen AH, Barbieri RL, et al. (2004) Endogenous estrogen, androgen, and progesterone concentrations and breast cancer risk among postmenopausal women. $J$ Natl Cancer Inst 96, 1856-1865.

5. Gruber CJ, Tschugguel W, Schneeberger C, et al. (2002) Production and actions of estrogens. N Engl J Med 346, 340-352.

6. Al-Azzawi F \& Palacios S (2009) Hormonal changes during menopause. Maturitas 63, 135-137.

7. World Cancer Research Fund \& American Institute for Cancer Research (2007) Food, Nutrition, Physical Activity, and Prevention of Cancer: A Global Perspective. Washington, DC: AICR.

8. Brinkman MT, Baglietto L, Krishnan K, et al. (2010) Consumption of animal products, their nutrient components and postmenopausal circulating steroid hormone concentrations. Eur J Clin Nutr 64, 176-183.

9. Holmes MD, Spiegelman D, Willett WC, et al. (2000) Dietary fat intake and endogenous sex steroid hormone levels in postmenopausal women. J Clin Oncol 18, 3668-3676.

10. Wu AH, Pike MC \& Stram DO (1999) Meta-analysis: dietary fat intake, serum estrogen levels, and the risk of breast cancer. J Natl Cancer Inst 91, 529-534.

11. Olson SH, Bandera E V \& Orlow I (2007) Variants in estrogen biosynthesis genes, sex steroid hormone levels, and endometrial cancer: a HuGE review. Am J Epidemiol 165, 235-245.

12. Hosono S, Ito H, Oze I, et al. (2015) Polymorphisms in CYP19A1, HSD17B1 and HSD17B2 genes and serum sex hormone level among postmenopausal Japanese women. Maturitas 82, 394-401.

13. Kidokoro K, Ino K, Hirose $\mathrm{K}$, et al. (2009) Association between CYP19A1 polymorphisms and sex hormones in postmenopausal Japanese women. J Hum Genet 54, 78-85.

14. Shi L, Yang X, Dong X, et al. (2016) Polymorphism of HSD17B1 Ser312Gly with cancer risk: evidence from 66,147 Subjects. Twin Res Hum Genet 19, 136-145.

15. Feigelson HS, Cox DG, Cann HM, et al. (2006) Haplotype analysis of the HSD17B1 gene and risk of breast cancer: a comprehensive approach to multicenter analyses of prospective cohort studies. Cancer Res 66, 2468-2475.

16. Nagata C, Nagao Y, Shibuya C, et al. (2005) Fat intake is associated with serum estrogen and androgen concentrations in postmenopausal Japanese women. J Nutr 135, 2862-2865.

17. Hamajima N (2007) The Japan Multi-Institutional Collaborative Cohort Study (J-MICC Study) to detect gene-environment interactions for cancer. Asian Pac J Cancer Prev 8, 317-323.

18. Wakai K, Hamajima N, Okada R, et al. (2011) Profile of participants and genotype distributions of 108 polymorphisms in a 
cross-sectional study of associations of genotypes with lifestyle and clinical factors: a project in the Japan Multi-Institutional Collaborative Cohort (J-MICC) Study. J Epidemiol 21, 223-235.

19. Tokudome S, Goto C, Imaeda N, et al. (2004) Development of a data-based short food frequency questionnaire for assessing nutrient intake by middle-aged Japanese. Asian Pac J Cancer Prev 5, 40-43.

20. Tokudome Y, Goto C, Imaeda N, et al. (2005) Relative validity of a short food frequency questionnaire for assessing nutrient intake versus three-day weighed diet records in middle-aged Japanese. J Epidemiol 15, 135-145.

21. Imaeda N, Goto C, Tokudome Y, et al. (2007) Reproducibility of a short food frequency questionnaire for Japanese general population. J Epidemiol 17, 100-107.

22. Resources Council, Science and Technology Agency, Japan (2000) Standard Tables of Food Composition in Japan, 5th ed. Tokyo: Resources Council, Science and Technology Agency, Japan (in Japanese).

23. Wakai K, Tamakoshi K, Date C, et al. (2005) Dietary intakes of fat and fatty acids and risk of breast cancer: a prospective study in Japan. Cancer Sci 96, 590-599.

24. Kuriki K, Hirose K, Wakai K, et al. (2007) Breast cancer risk and erythrocyte compositions of $n-3$ highly unsaturated fatty acids in Japanese. Int J Cancer 121, 377-385.

25. Zheng J-S, Hu X-J, Zhao Y-M, et al. (2013) Intake of fish and marine $n-3$ polyunsaturated fatty acids and risk of breast cancer: meta-analysis of data from 21 independent prospective cohort studies. BMJ 346, f3706.

26. Ohnishi Y, Tanaka T, Ozaki K, et al. (2001) A high-throughput SNP typing system for genome-wide association studies. J Hum Genet 46, 471-477.

27. Dallal CM, Tice JA, Buist DSM, et al. (2014) Estrogen metabolism and breast cancer risk among postmenopausal women: a casecohort study within B FIT. Carcinogenesis 35, 346-355.

28. Falk RT, Brinton LA, Dorgan JF, et al. (2013) Relationship of serum estrogens and estrogen metabolites to postmenopausal breast cancer risk: a nested case-control study. Breast Cancer Res 15, R34.

29. Fuhrman BJ, Schairer C, Gail MH, et al. (2012) Estrogen metabolism and risk of breast cancer in postmenopausal women. J Natl Cancer Inst 104, 326-339.

30. Key TJ, Appleby PN, Reeves GK, et al. (2015) Steroid hormone measurements from different types of assays in relation to body mass index and breast cancer risk in postmenopausal women: reanalysis of eighteen prospective studies. Steroids 99, 49-55.

31. Nagata C, Nagao Y, Yamamoto S, et al. (2008) Light exposure at night, urinary 6-sulfatoxymelatonin, and serum estrogens and androgens in postmenopausal Japanese women. Cancer Epidemiol Biomarkers Prev 17, 1418-1423.

32. Campbell KL, Foster-Schubert KE, Alfano CM, et al. (2012) Reduced-calorie dietary weight loss, exercise, and sex hormones in postmenopausal women: randomized controlled trial. J Clin Oncol 30, 2314-2326.

33. van Gemert WAM, Schuit AJ, van der Palen J, et al. (2015) Effect of weight loss, with or without exercise, on body composition and sex hormones in postmenopausal women: the SHAPE-2 trial. Breast Cancer Res 17, 120.

34. Murtaugh MA, Herrick J, Sweeney C, et al. (2011) Macronutrient composition influence on breast cancer risk in Hispanic and non-Hispanic white women: the 4-Corners Breast Cancer Study. Nutr Cancer 63, 185-195.

35. Lajous M, Boutron-Ruault M-C, Fabre A, et al. (2008) Carbohydrate intake, glycemic index, glycemic load, and risk of postmenopausal breast cancer in a prospective study of French women. Am J Clin Nutr 87, 1384-1391.
36. Sugawara N, Yasui-Furukori N, Umeda T, et al. (2015) Relationship between dietary patterns and cognitive function in a community-dwelling population in Japan. Asia Pac J Public Health 27, NP2651-NP2660.

37. Wu AH, Stanczyk FZ, Seow A, et al. (2002) Soy intake and other lifestyle determinants of serum estrogen levels among postmenopausal Chinese women in Singapore. Cancer Epidemiol Biomarkers Prev 11, 844-851.

38. Smith-Warner SA, Spiegelman D, Adami HO, et al. (2001) Types of dietary fat and breast cancer: a pooled analysis of cohort studies. Int J Cancer 92, 767-774.

39. Tokudome Y, Imaeda N, Ikeda M, et al. (1999) Foods contributing to absolute intake and variance in intake of fat, fatty acids and cholesterol in middle-aged Japanese. J Epidemiol $\mathbf{9}$, 78-90.

40. Kuriki K, Nagaya T, Tokudome Y, et al. (2003) Plasma concentrations of $(n-3)$ highly unsaturated fatty acids are good biomarkers of relative dietary fatty acid intakes: a crosssectional study. J Nutr 133, 3643-3650.

41. Goto C, Tokudome Y, Imaeda N, et al. (2006) Validation study of fatty acid consumption assessed with a short food frequency questionnaire against plasma concentration in middle-aged Japanese people. Food Nutr Res 50, 77-82.

42. Young LR, Kurzer MS, Thomas W, et al. (2011) Effect of dietary fat and omega-3 fatty acids on urinary eicosanoids and sex hormone concentrations in postmenopausal women: a randomized controlled feeding trial. Nutr Cancer 63, 930-939.

43. Larsson SC, Kumlin M, Ingelman-Sundberg M, et al. (2004) Dietary long-chain $n-3$ fatty acids for the prevention of cancer: a review of potential mechanisms. Am J Clin Nutr 79, 935-945.

44. Richards JA \& Brueggemeier RW (2003) Prostaglandin E2 regulates aromatase activity and expression in human adipose stromal cells via two distinct receptor subtypes. J Clin Endocrinol Metab 88, 2810-2816.

45. Puranen TJ, Poutanen MH, Peltoketo HE, et al. (1994) Sitedirected mutagenesis of the putative active site of human 17 betahydroxysteroid dehydrogenase type 1. Biochem J 304, 289-293.

46. The National Center for Biotechnology Information (2016) Cluster Report: rs605059. http://www.ncbi.nlm.nih.gov/ projects/SNP/snp_ref.cgi?rs=605059 (accessed July 2016).

47. Wu AH, Seow A, Arakawa K, et al. (2003) HSD17B1 and CYP17 polymorphisms and breast cancer risk among Chinese women in Singapore. Int J Cancer 104, 450-457.

48. Iwasaki M, Hamada GS, Nishimoto IN, et al. (2010) Dietary isoflavone intake, polymorphisms in the CYP17, CYP19, 17beta-HSD1, and SHBG genes, and risk of breast cancer in case-control studies in Japanese, Japanese Brazilians, and non-Japanese Brazilians. Nutr Cancer 62, 466-475.

49. Setiawan VW, Hankinson SE, Colditz GA, et al. (2004) HSD17B1 gene polymorphisms and risk of endometrial and breast cancer. Cancer Epidemiol Biomarkers Prev 13, 213-219.

50. Yao L, Cao L-H, Qiu L-X, et al. (2010) The association between HSD17B1 Ser312Gly polymorphism and breast cancer risk: a meta-analysis including 31,053 subjects. Breast Cancer Res Treat 123, 577-580.

51. Date C, Fukui M, Yamamoto A, et al. (2005) Reproducibility and validity of a self-administered food frequency questionnaire used in the JACC study. J. Epidemiol 15, Suppl. 1, S9-S23.

52. Tsugane S, Kobayashi M, Sasaki S, et al. (2003) Validity of the self-administered food frequency questionnaire used in the 5-year follow-up survey of the JPHC Study Cohort I: comparison with dietary records for main nutrients. J Epidemiol 13, S51-S56.

53. Karim R, Stanczyk FZ, Brinton RD, et al. (2015) Association of endogenous sex hormones with adipokines and ghrelin in postmenopausal women. J Clin Endocrinol Metab 100, 508-515. 